anti-dsDNA antibodies $(\mathrm{Ab})$ and the modulation of the spliceosome in the SLE monocytes activity.

Methods: Sixty SLE patients and forty healthy donors (HD) were included in the study. Infiltration rate of myeloid cells and its association with clinical features were analyzed in kidney biopsies by Immunohistochemistry. In parallel, circulating monocytes were purified from peripheral blood by immune-magnetic selection. The expression of a set of 770 genes related to autoimmune/inflammatory diseases was evaluated using NanoString Technologies. The levels of the main 45 components of the splicing machinery were further analyzed in these samples using a microfluidic qPCR array (Fluidigm). An extensive clinical/serological evaluation was also performed, comprising disease activity, renal involvement parameters, autoAb profile, and the systemic inflammatory status (27-plex Assay). Finally, in vitro studies involving anti-dsDNA-IgG Ab treatment and over/down-expression of splicing machinery components were carried out to analyze their effects in the monocyte activity. Results: Infiltration of CD68 expressing cells was confirmed in kidney biopsies and associated with parameters of kidney failure (C3/C4, chronic index), highlighting the key role of the myeloid compartment in lupus nephropathy. Gene expression profiling recognized 156 genes differentially expressed in SLE monocytes compared with HDs, including 87 genes up-regulated and 69 down-regulated. Functional analysis showed that most dysregulated genes were associated with the IFN response (i.e. IFIT1, IFI44, IFI44L, RSAD2). In parallel, the altered expression of 27 spliceosome components was demonstrated in SLE monocytes compared with HD, including 3 up-regulated and 24 down-regulated. Correlation studies demonstrated that the aberrant expression of splicing machinery components was linked to the altered interferon signature and the plasma inflammatory profile. This aberrant profile at molecular level was associated with the disease activity status, anti-dsDNA positivity and C3/C4 levels. Interestingly, SLE patients with renal disease displayed a simultaneous alteration of both, the IFN and the spliceosome signatures in monocytes, along with an enlarged pro-inflammatory profile in plasma. Logistic regression models that integrated the concomitant alteration of some splicing machinery components and IFNs genes identified lupus nephritis patients with high accuracy. Mechanistic studies showed that in vitro treatment of monocytes from HDs with anti-dsDNA promoted a concomitant deregulation of the IFN signature and the expression of several spliceosome components (i.e. PTB, RBM17, RNU6ATAC). Finally, the over/down-expression of selected spliceosome components (PTB and RBM17) in monocytes from SLE patients reduced the active release of inflammatory cytokines and their adhesion capacity.

Conclusion: 1) Monocytes from SLE patients with renal involvement exhibit a remarkable alteration of genes associated with the IFN response, further linked with the aberrant expression of several splicing machinery components. 2) Anti-dsDNA promoted the dysregulation in monocytes of both the IFN and spliceosome signatures, along with an active release of pro-inflammatory mediators. 3) The modulation of key splicing components in monocytes from SLE patients reduce their pro-inflammatory status and migration capacity. Ongoing studies may provide novel biomarkers and therapeutic tools to treat lupus nephropathy.

Acknowledgements: Funded by ISCIII, PI18/00837 and RIER RD16/0012/0015 co-funded with FEDER

Disclosure of Interests: None declared

DOI: 10.1136/annrheumdis-2021-eular.2534

\section{POS0420 \\ DYSREGULATED CD38 EXPRESSION ON PERIPHERAL BLOOD IMMUNE CELL SUBSETS IN SLE}

M. Burns ${ }^{1}$, L. Ostendorf ${ }^{2,3}$, A. Grützkau ${ }^{1}$, F. Hiepe ${ }^{2,3}$, T. Alexander ${ }^{2,3}$, H. Mei ${ }^{1}$. ${ }^{1}$ Deutsches Rheuma-Forschungszentrum (DRFZ Berlin) - a Leibniz Institute, Immune Monitoring \& Mass Cytometry, Berlin, Germany; ${ }^{2}$ Charité - Universitätsmedizin Berlin, Department of Rheumatology and Clinical Immunology, Berlin, Germany; ${ }^{3}$ Deutsches Rheuma-Forschungszentrum (DRFZ Berlin) - a Leibniz Institute, Autoimmunology, Berlin, Germany

Background: Systemic lupus erythematosus (SLE) is a chronic autoimmune disease characterized by pathogenic antinuclear autoantibodies, which are secreted by autoreactive plasma cells. Among novel plasma cell-depleting strategies, CD38 has been identified as promising target. The monoclonal anti-CD38 antibody daratumumab is approved for treatment of multiple myeloma and provided a therapeutically relevant depletion of plasma cells in patients with SLE'

Objectives: Beyond plasma cells, CD38 is widely expressed across innate and adaptive immune cells and additional cellular targets of anti-CD38 treatment, especially in patients with SLE, are largely unknown. Therefore, this study aimed to systematically characterize the expression of CD38 in peripheral blood leukocytes to identify potential target cells of CD38-directed therapies that may contribute to or limit therapeutic benefits in SLE.

Methods: We analyzed the expression of CD38 on peripheral blood leukocytes in two different cohorts, comprising a total of 56 SLE patients and 39 healthy controls, by flow and mass cytometry. CD38 expression levels across major immune cells were analyzed for changes between controls and SLE, as well as for correlation across immune cell lineages, and with clinical and serological disease parameters.
Results: We detected increased CD38 expression levels on circulating NK cells, plasmacytoid dendritic cells, $\mathrm{CD}^{+}$and $\mathrm{CD}^{+}$memory T cells, as well as $\lg \mathrm{D}^{\circ} \mathrm{CD} 27^{-}$and marginal zone-like B cells in SLE compared to healthy controls. In myeloid and NK cells, CD38 expression was associated with an activated cellular phenotype, reflected by co-expression of molecules such as HLA-DR, CD11c or Syk. In the B cell compartment, IgA- plasmablasts and plasma cells expressed more CD38 than their $\operatorname{lgA}^{+}$counterparts. Also, HLA-DR ${ }^{\text {high }}$ plasmablasts showed higher CD38 expression compared to HLA-DR ${ }^{\text {low }}$ plasma cells. The strongest differences in CD38 expression between controls and SLE were found in $\mathrm{CD}^{+}$central and effector memory $\mathrm{T}$ cells. Additionally, we detected an expansion in CD38 ${ }^{\text {high }}$ and $C D 38^{\text {int }}$ cells in the $T$ cell memory compartment, with some patients showing distinctly increased expression values. We observed a high intra-individual correlation of CD38 expression across immune cell lineages, yet without correlation of CD38 expression levels with clinica activity (SLEDAI-2K), serological markers of SLE or the type I interferon surrogate marker CD169 (SIGLEC-1)

Conclusion: Our data indicate that not only pathogenic plasma cells are potential target cells of CD38-targeting antibodies. The highly dysregulated CD38 expression across innate and adaptive immune cells in SLE could be of pathophysiological importance with respect to the potential efficacy and side effects of such therapies. Since CD38 expression did not correlate with disease activity, it may be assumed that it is not a response protein solely induced and modulated by type I interferons. Nevertheless, our comprehensive characterization of CD38 expression in the immune system might have important implications for personalized approaches with emerging CD38-directed therapeutics.

\section{REFERENCES:}

[1] Ostendorf, L. et al. Targeting CD38 with Daratumumab in Refractory Systemic Lupus Erythematosus. N. Engl. J. Med. 383, 1149-1155 (2020).

Disclosure of Interests: None declared

DOI: 10.1136/annrheumdis-2021-eular.3883

\section{POS0421 COMBINED ANALYSIS OF METABOLIC AND TRANSCRIPTOMIC KIDNEY PROFILES OF NZW/ B-F1 MURINE LUPUS UNCOVERS BIOLOGICAL MECHANISMS PRECEDING THE ONSET OF NEPHRITIS}

T. Manolakou $^{1}$, I. Tsiara ${ }^{2}$, D. Nikolopoulos ${ }^{1,3}$, P. Garantziotis ${ }^{1,4}$, D. Benaki ${ }^{2}$ E. Gikas ${ }^{2}$, E. Frangou ${ }^{1,5}$, E. Mikroa ${ }^{2}$, D. Boumpas ${ }^{1,3} .{ }^{1}$ Biomedical Research Foundation of the Academy of Athens, Center of Translational Research, Athens, Greece; ${ }^{2}$ National and Kapodistrian University of Athens, Department of Pharmacy, Section of Pharmaceutical Chemistry, Athens, Greece; ${ }^{3}$ National and Kapodistrian University of Athens, Rheumatology and Clinical Immunology Unit, 4th Department of Internal Medicine, Athens, Greece; ${ }^{4}$ Hannover Medical University, Division of Immunology and Rheumatology, Hannover, Germany; ${ }^{5}$ Limassol General Hospital, Department of Nephrology, Limassol, Cyprus

Background: Metabolic pathways are important regulators of immune differentiation and activation in kidneys. Kidneys directly impact systemic metabolism, circulating metabolite levels, and express intrinsic metabolic activity. The integration of renal metabolomic and transcriptomic profiles may unravel unique gene-metabolite pairs of biological significance in lupus nephritis (LN)

Objectives: To decipher gene-metabolite signatures at both pre-nephritic and nephritic stages of lupus.

Methods: Kidneys were isolated and snap-frozen after perfusion from female $\mathrm{NZB} / \mathrm{NZW}-\mathrm{F} 1$ lupus mice at the pre-nephritic (3-month-old) and nephritic (6-month-old exhibiting $\geq 100 \mathrm{ng} / \mathrm{dL}$ of urine protein) stage of lupus ( $\mathrm{n}=6 / \mathrm{group}$ ). Age-matched female C57BL/6 mice were used as healthy controls. Sample extracts were used for RNA sequencing and ${ }^{1} \mathrm{H}-\mathrm{NMR}$ spectroscopy metabolic profiling. DESeq2 was used to identify differentially expressed genes. Univariate analysis was used to reveal metabolic differences characteristic for nephritis.

Results: Comparative transcriptomic analyses uncovered multiple transcripts related to metabolic pathways: In pre-nephritic kidneys, lipid metabolism, cellular respiration, TCA cycle, amino acid metabolism processes were overrepresented in the upregulated genes while in nephritic kidneys, amino acid metabolism processes were overrepresented among the downregulated genes (Figure 1). ${ }^{1} \mathrm{H}-\mathrm{NMR}$ analysis revealed a total of 49 metabolites. Comparison of the metabolic levels of nephritic and pre-nephritic animals revealed that ADP, ATP, NAD+, Taurine and Myo-inositol decreased, while Thr increased significantly. The comparison to corresponding control animals, demonstrated that only myo-inositol increased significantly. Integration of kidney metabolomics and transcriptomics indicated the involvement of processes related to glutathione metabolism, leukocyte trans-endothelial migration and antigen presentation during the established renal disease stage.

Conclusion: The combined transcriptomics and metabolomics analysis revealed metabolic derangements in lupus-affected kidneys both during subclinical and overt LN. Deregulated tissue-levels of taurine and myo-inositol at the subclinical stage of the disease suggest aberrant renal biochemistry preceding the development of overt LN that may directly impact systemic metabolism and circulating metabolite levels. 